\title{
The Jiles Atherton Model for Description Of Hysteresis in Lithium Battery
}

\author{
Marco Trapanese \\ Dipartimento di Ingegneria Elettrica, Elettronica, delle \\ Telecomunicazioni, Chimica, Automazione e Matematica \\ Palermo University \\ Palermo, Italy \\ marco.trapanese@unipa.it
}

\author{
Vincenzo Franzitta, Alessia Viola \\ Dipartimento dell' Energia \\ Palermo University \\ Palermo, Italy \\ franzitta@dream.unipa.it \\ alessia.viola@unipa.it
}

\begin{abstract}
In this paper Jiles Atherton (JA) Model is used to obtain a mathematical model of the hysteresis in lithium battery. JA Model allows to describe both the hysteresis and the dynamical features of charging and discharging cycles in a lithium battery. The identification of the model is obtained by using a neural network technique developed for magnetic systems. The model is validated on some experimental tests on commercial batteries.
\end{abstract}

\section{INTRODUCTION}

The main purpose of this paper is to adapt Jiles Atherton (JA) model of hysteresis in magnetic material to the description of hysteresis in Lithium battery. JA model is a powerful mathematical tool developed to describe hysteresis in magnetic materials and in this field this model is considered reliable and accurate. It is well known that a reliable mathematical model of batteries is a requisite for a full exploitation of their potentiality in order to develop complex electrical systems. The mathematical model needed varies greatly and its complexity and accuracy is strongly related to the type of application in which the model is required. In applications where the current is roughly constant the mathematical model needed is quite simple, but in systems like cars, tools or smart grid the working condition varies greatly and therefore more sophisticated tools are needed. Especially in these systems it is very important to be able to describe and foresee the dynamic features of the chargingdischarging cycles. Therefore, the estimation of the state of charge (SOC) of the battery is a key task in order to describe its behavior. The description of hysteresis features in batteries has been attempted with several approaches, for example in some papers the model developed uses the concept of equivalent circuit with some parameters added to improve the accuracy [1], in others the classical Preisach model is used [26]. Classical Preisach Model is a powerful tool to describe static hysteresis but it is not able to grasp the dynamic features of hysteresis phenomenon [3]. However a detailed model of the dynamic feature is very important in many applications and can help to overcome several limitation in the use of batteries [7-15] In this paper, JA Model, is adapted and used to obtain the dynamical features of hysteresis in lithium battery. This digest preliminarily introduces JA Model and illustrates the adaptation needed to permit its application to Lithium battery. Then, a identification procedure of the model based on Neural Network is presented, finally the model is validated on some experimental results.

\section{JA MODEL}

The J-A model has the following form:

$$
\frac{d S}{d H}=\frac{(1-c) \frac{d S_{i r r}}{d H_{e}}+c \frac{d S_{a n}}{d H_{e}}}{1-\alpha c \frac{d S_{a n}}{d H_{e}}-\alpha(1-c) \frac{d S_{i r r}}{d H_{e}}}
$$

Where: $\mathrm{S}$ is the state of charge, $\mathrm{H}$ is the applied voltage, $\mathrm{c}$ is the coefficient of reversibility of the battery, Sirr is the irreversibile Sate of charge, He is the effective voltage, San is the anhysteretic state of charge, $\alpha$ is linked to hysteresis losses.

$\mathrm{S}_{\mathrm{an}}$ can be calculated through the Langevin's equation:

$$
S_{a n}\left(H_{e}\right)=M_{s}\left(\operatorname{coth}\left(\frac{H_{e}}{a}-\frac{a}{H_{e}}\right)\right)
$$

where $S_{s}$ is the saturation state of charge, $a$ is a form factor and $\mathrm{S}_{\text {irr }}$ is defined as follows:

$$
\frac{d S_{i r r}}{d H_{e}}=\operatorname{sign}\left(\frac{d H}{d t}\right) \frac{M_{a n}-S_{i r r}}{k}
$$


where $\mathrm{t}$ is time and $\mathrm{k}$ is a numerical constant which depends on the type of battery. As a result, J-A model is a five-parameter model.

\section{THE IDENTIFICATION PROCEDURE}

The identification procedure consists of three steps: A) training; B) submission of data; C) output. The step $\mathrm{A}$ is executed separately from steps $\mathrm{B}$ and $\mathrm{C}$

\section{A. Training}

Training phase aims to build a network, whose input is the hysteresis curve and the output consists of the parameters of J-A Model that better reproduces the hysteresis cycle loop which had been submitted to the network.

The neural network used consists of a three layer neural network: the first layer consists of 25 linear neurons, the second layer of 40 neurons with hyperbolic tangent sigmoid function, and the output layer of 5 linear neurons. The input is made up of a 32-component vector. The first layer makes a linear reduction from the high dimension input space to a slightly lower 25-dimension space. The output of the first layer is therefore the feature vector that sums up the most relevant characteristics of the input space and is strictly connected to five relevant properties of the hysteresis loop. The 40 neurons of the second layer neurons allows to store a number of parametric curves equal at least equal to 1000 . The output layer is made up of 5 neurons which are the free parameters of J-A model that most suitably permits to produce the inputs. In Fig. 1 it is shown shows how the network works.

The training set is made of 243 vectors. Each input vector has 32 coordinates, while the target vectors are defined on a 5D space. The 32 coordinates are the ordinates of a first order reversal hysteresis curve obtained under sinusoidal excitation. The excitation amplitude is the same in all cases and all the transients have died out. Each input vector was computed by inserting in eqs 1,2 and 3 a unique set of parameters.

In the end The the Levenberg-Marquadt algorithm has been used for the training phase

\section{B. Submission of Data.}

Each input vector belongs to a 32D space and is made up of the the ordinates of a hysteresis loop. Hysteresis loop data must spans the same interval spanned from the vectors used in the training phase.

\section{Output of the Results}

The output of the network consists of five parameters. They represent the parameters of the J-A model that is associated to the presented loop. These numbers are the interpolation of the network to the best value that it is able to output to describe the presented hysteresis loop.

\section{EXPERIMENTAL RESULTS}

In order to verify the ability of the model to describe the dynamic behavior of charging discharging cycles, in this digest a 1 A charging discharging cycle is shown in fig. 1 . The cycle applied to the battery has the same features of one of those reported in [1]. The experimental cycle was submitted to a previously trained network which gave as output the five parameters to be used in the JA Model to reconstruct the cycle. Fig. 1 shows how the proposed approach is able to describe the dynamic features of the cycle. The error is within $5 \%$. In the paper several other cycles will be shown.

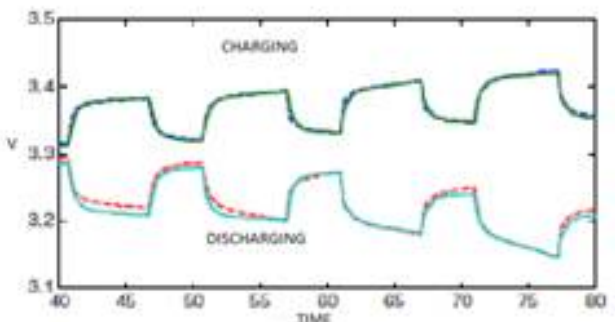

Figure 1. A 1 1A charging discharging cycle. The solid line is the experimental result the dashed one is the mathematical reconstruction given by JA Model

\section{CONCLUSION AND FUTURE WORK}

The work reported in this digest shows how JA can be a useful mathematical tool to describe the dynamic behavior of Lithium battery. In the paper several other experimental evidences will be given and a real time SOC strategy based on JA will be presented.

\section{ACKNOWLEDGMENT}

This work was supported under project "Impetus" by Ministero dell'Ambiente and by MIUR (Ministero dell'Istruzione e dell'Università) of Italian Republic.

\section{REFERENCES}

[1] Ala Al-Haj Hussein, Nasser Kutkut, Issa Batarseh, "A Hysteresis Model for a Lithium Battery Cell with Improved Transient Response," in IEEE 2011 Applied Power Electronics Conference and Exposition (APEC), 2011, pp.1790-1794.

[2] Xidong Tang, Xiaodong Zhang, Brian Koch, and Damon Frisch, "Modeling and Estimation of Nickel Metal Hydride Battery Hysteresis for SOC Estimation," in IEEE 2008 International Conference on Prognostics and Health Managment, 2008, pp.1-12.

[3] Trapanese Marco, "Identification of parameters of dynamic Preisach model by neural networks ", Journal of Applied Physics, Volume: 103 , Issue: 7, 2008 , pp. 07D929 - 07D929-3.

[4] Franzitta, V., Rizzo, G.. - Renewable energy sources: A mediterranean perspective. ICBEE 2010 - 2nd International Conference on Chemical, Biological and Environmental Engineering, Proceedings, p.48-51, art. no. 5652332 (2010).

[5] Bonanno, A., Franzitta, V., Muzio, F.P., Trapanese, M. - A multiphysics approach to the design of a seawave energy conversion system. Proceedings of ICSET, Singapore, 2008, p.p. $665-668$, art. no. 4747090 .

[6] Di Dio, V., Franzitta, V., Muzio, F., Scaccianoce, G., Trapanese, M. The use of sea waves for generation of electrical energy and hydrogen. MTS/IEEE Biloxi - Marine Technology for Our Future: Global and Local Challenges, OCEANS, 2009, art. no. 5422319.

[7] Franzitta, V., La Gennusa, M., Peri, G., Rizzo, G., Scaccianoce, G. Toward a European Eco-label brand for residential buildings: Holistic or by-components approaches?. Energy 2011; 36(4 ), 1884-1892. 
[8] Sorrentino, G., Scaccianoce, G., Morale, M., Franzitta, V. - The importance of reliable climatic data in the energy evaluation. Energy 2012.

[9] Trapanese, M., Viola, A., Franzitta, V. - Description of hysteresis of nickel metal hybride battery. IECON 2012, 38th Annual Conference on IEEE Industrial Electronics Society, Montreal, Canada, pp. 967-970.

[10] Ciulla, G., Franzitta, V., Lo Brano, V., Viola, A., Trapanese, M. - Mini Wind Plant to Power Telecommunication Systems: a Case Study in Sicily - Advanced Materials Research Vols. 622-623 (2013) pp 10781083 .

[11] Sorrentino, G., Ferrante, P., Franzitta, V., La Gennusa, M., Nicolosi, S., Scaccianoce, G., Viola, A. - Generation of a Test Reference Year (TRY): an application to the town of Palermo - Advanced Materials Research Vols. 622-623 (2013) pp 1835-1840.
[12] Trapanese, M., Viola, A., Franzitta, V. - Description of hysteresis in Lithium battery by classical Preisach model - Advanced Materials Research Vols. 622-623 (2013) pp 1099-1103.

[13] Trapanese, M., Viola, A., Franzitta, V. - Design And Experimental Test Of A Thermomagnetic Motor - Aasri Procedia 2 (2012) 199 - 204

[14] Trapanese, M., Viola, A., Franzitta, V. - Seawave Power Farm Design: A Case Study - AASRI Procedia 2 (2012) 223 - 228.

[15] Ciulla, G., Franzitta, V., Lo Brano, V., Viola, A. - A Novel implicit Correlation for the Operative Temperature of a PV Panel - AASRI Procedia 2 (2012) 112-120 